This is the accepted version of the article:

Zinoviev, K.E.; Gonzalez-Guerrero, A.B.; Dominguez, C.; Lechuga, L.M.. Integrated Bimodal Waveguide Interferometric Biosensor for Label-Free Analysis. J ournal of Lightwave Technology, (2011). 29. 13: 1926 - . 10.1109/J LT.2011.2150734.

Available at: https://dx.doi.org/10.1109/J LT.2011.2150734 


\title{
Integrated Bimodal Waveguide Interferometric Biosensor for Label-free Analysis
}

\author{
Kirill E. Zinoviev, Ana Belén González-Guerrero, Carlos Domínguez, Laura M. Lechuga
}

\begin{abstract}
The performance of an interferometric device based on integrated Bimodal Waveguides (BiMW) for sensing is demonstrated. The sensors are fabricated using standard silicon technology and can achieve a detection limit of $2.5 \cdot 10^{-7}$ RIU for homogeneous sensing, rendering in a very high sensitive device. The applicability of the bimodal waveguide interferometer as label-free biosensor has been demonstrated by the real-time monitoring of the biomolecular interaction of BSA and anti-BSA. Due to their simplicity, the interferometric devices could be further integrated in complete lab-on-a-chip platforms for point-of-care diagnostics showing them as a powerful instrument for biochemical analysis.
\end{abstract}

Index Terms - Bimodal waveguide, optical biosensors, integrated optics, evanescent wave detection, waveguides

\section{INTRODUCTION}

$\mathrm{M}$ OST diagnostics tests are based on time-consuming, expensive, and sophisticated techniques performed by specialized technicians in laboratory environments. These techniques typically require labeling of the samples or reagents with fluorescent or radioactive tags. There is an unmet need of having reliable diagnostic tools that ensure a sensitive, rapid, affordable and simple analysis, particularly in the clinical practice. Such reliable diagnostic tools could afford the decentralization of diagnostics (clinical or environmental) to point-of-care (POC) settings, allowing tests in workplaces, homes or primary care facilities, among others.

Last advances in micro- and nanosensing technology are offering the implementation of diagnostic tools with increased sensitivity, specificity, and reliability for in vivo and in vitro

Manuscript received January 24, 2011. This work was supported by Fundación M. Botín, SENA project (TSI-020301-2008-11) from the Spanish Ministry of Industry and LEUKEMCHIP project (Internal program, CIBER$\mathrm{BBN})$

K. E. Zinoviev and C. Domínguez are with Micro and Nanosystems Department, at Instituto de Microelectronica de Barcelona, CNM (CSIC) Campus UAB, 08193, Cerdanyola del Valles, Barcelona, Spain. (corresponding author, phone: +34-93-594-7700; fax: +34-93-586-1496; email: kirill.zinoviev@imb-cnm.csic.es).

Ana Belen Gonzalez-Guerrero, Laura M. Lechuga are with Nanobiosensors and Bioanalytical Applications Group, CIN2 (CSIC-ICN) and CIBER-BBN, Campus UAB, 08193, Cerdanyola del Valles, Barcelona, Spain.

Copyright (c) 2011 IEEE. Personal use of this material is permitted However, permission to use this material for any other purposes must be obtained from the IEEE by sending a request to pubs-permissions@ieee.org. applications. The application of a portable, easy-to-use and highly sensitive biosensor lab-on-a-chip platform for real-time diagnosis could offer significant advantages over current methods [1]. Photonic biosensors based on evanescent wave detection are one of the best candidates as detectors in such platform due to their high sensitivity.

Among evanescent wave optical biosensors, interferometric sensors are recognized to be one of the most sensitive devices for label-free analysis. The sensitivity to homogeneous changes in the refractive index can reach $10^{-8}$ of refractive index unit (RIU) which is at least two orders of magnitude better than, for example, the sensitivity of Surface Plasmon Resonance (SPR) sensors [2]. But problems of stability, complex manipulation and read-out have prevented the general use of integrated interferometers biosensors for real field applications.

Integrated interferometric sensors have been extensively studied over the last two decades. The configurations based on Mach Zehnder (MZI) [1] and Young (YI) [3] interferometers are the most well-known. Both use a waveguide which is splitter by using a Y-junction in two waveguide channels, one for sensing and one for reference. The main difference between them is that the interference pattern readout is on chip in the case of the MZI (the two channels are recombined again by a second Y-junction) and off chip in the case of the YI. MZI interferometers have the advantage of simple signal readout using a single photodetector, but they have the disadvantage of requiring high stability for light coupling, which is the main bottleneck for their practical implementation. In the Young configuration, the relative changes in the interference pattern are measured in far field. They are almost independent on the light intensity, making them more reliable and easier to use. There is another type of interferometric sensor, i.e. differential interferometer (DI) [4] in which the propagation of two orthogonal polarizations in a unique single-mode waveguide is analyzed. Two orthogonal polarizations are also used in a technique based on a mixture of YI and DI methods, called Dual Polarization Interferometry (DPI) [5]. The DPI sensor allows simultaneous measuring of thickness and refractive index of a film adsorbed on the sensor surface. In all the above described interferometric sensors the design of the integrated sensor includes complex components (as splitters) and/or the instrumentation is bulky, making difficult to achieve a truly lab-on-a-chip portable device.

We present here the results of a single channel waveguide interferometer operated on interference of two waveguide 
modes (fundamental and first order modes) of the same polarization. The simplicity of the design of the bimodal waveguide (BiMW) interferometers is attractive for massfabrication as there is no need anymore of including Y-shape splitters, which are the most complex component of MZI and YI devices, responsible for the modulation depth of the output signal and, subsequently, for the sensitivity. The sensitivity evaluation of the device for both homogeneous and surface sensing have demonstrated the excellent performance of this new device rendering it as one of the most promising integrated optics-based biosensors for multiplexing analysis.

\section{BIMODAL WAVEGUIDE INTERFEROMETER: PRINCIPLE OF OPERATION}

The devices dealing with modal interference employ a single waveguide supporting the interfering modes [6-10]. The sketch of the device is shown in Fig.1. Light from a coherent source in TE polarization is coupled into a ridge waveguide supporting a single transversal mode. After some distance this mode is coupled into another waveguide which supports two transversal modes. Due to the vertical asymmetry of the junction, the fundamental mode of the first waveguide splits in two, the fundamental and the first order modes, which are propagating till the output of the chip. The modes propagate with different velocities and create an interference pattern at the exit, which intensity distribution depends on the physical parameters of the structure (width, thickness, rib height) and, in particular, on the refractive index of the cladding layer through the interaction with the evanescent field. This intensity distribution is monitored off chip using a photodetector array [9].

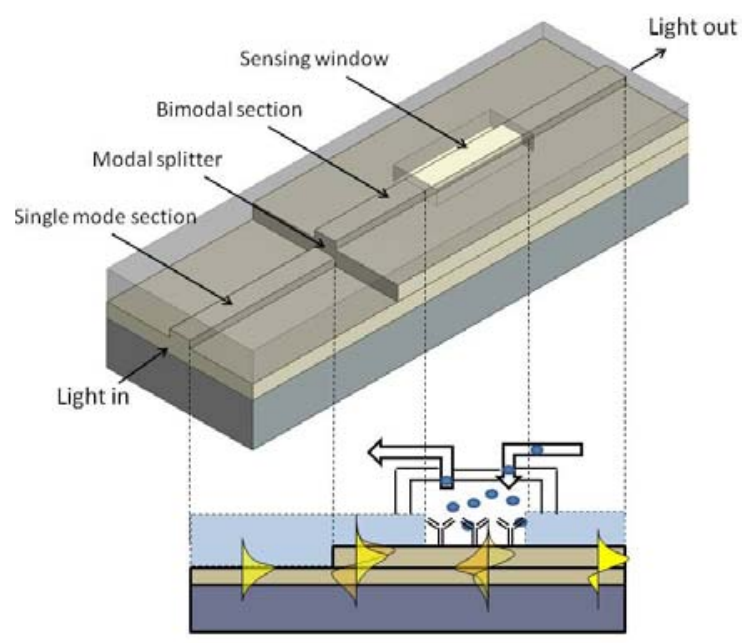

Fig. 1. Sketch of the BiMW device. Inset below: distribution of the electromagnetic field in several points through the waveguide and interaction of the evanescent wave with molecules for surface sensing.

For evaluation of the BiMW device, a light source with a narrow spectral line, stable in time, and with a coherency length of several millimeters is recommended for generating high contrast interference at the output of the BiMW. The BiMW device has an intrinsic sensitivity to temperature changes, because the propagation constants of both modes are differently affected by temperature changes. Taking advantage of this, temperature changes has been used for initial adjustment of the interference pattern and for preliminary characterization of the chip (sees Fig. 2). Using a temperature change, the modulation depth of the output signal and the speed of modulation can be observed, which can help in the estimation of the quality of the modal splitter and the bimodal part of the waveguide. The interference pattern is independent on the light intensity and the output signal is not sensitive to fluctuations in the coupling efficiency, which is an advantage as compared to classical integrated MZI devices which employ a $3 \mathrm{~dB}$ splitter and combiner fabricated as $\mathrm{Y}$-shape junctions [1]. In an integrated MZI fluctuations in the light intensity or in the coupling are transferred to the output signal and can be falsely interpreted as a result of a monitored reaction.
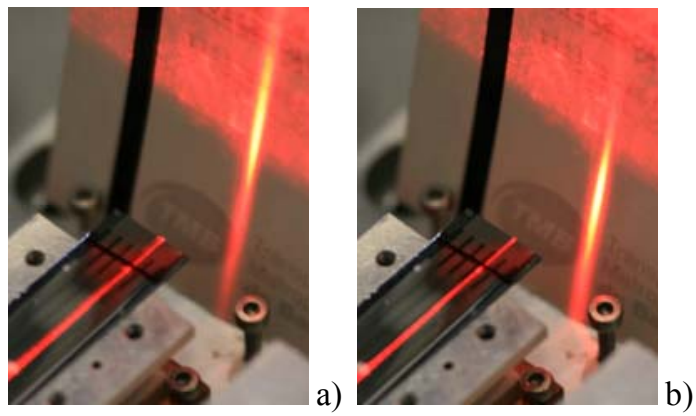

Fig. 2. Photographs of the light exiting the bimodal waveguide: the intensity is moving up (a) or down (b) as a function of the intermodal phase difference. Intermodal phase difference was achieved by varying chip temperature $0.5^{\circ} \mathrm{C}$.

\section{EXPERIMENTAL}

\section{A. The chip}

The devices were fabricated using standard microelectronics technology in our Clean Room facilities. A thermally grown silicon dioxide cladding layer (1.46 refractive index, $2 \mu \mathrm{m}$ thick) was deposited over a silicon wafer. A core layer of silicon nitride (2.00 refractive index) of $350 \mathrm{~nm}$ thickness was deposited by LPCVD technique. A ridge channel waveguide of $3 \mu \mathrm{m}$ width and with a ridge height of $2 \mathrm{~nm}$ was generated by BHF etching through a photoresist mask patterned by conventional photolithography.

The thickness of the single mode part of the waveguide was decreased to $150 \mathrm{~nm}$ using conventional photolithography and dry etching. This thickness was selected by simulations which indicate that for obtaining more than $70 \%$ modulation, the single mode part must have a thickness below $160 \mathrm{~nm}$. In the modeling, we used a transfer matrix approach for simulating the profiles of the electric fields of the modes in both single and bimodal areas. Then we used overlap integrals to estimate the coupling efficiency from the fundamental mode of the single mode part to the fundamental and first order modes of the bimodal part. Finally, by integrating the intensities of the fields at the exit over the upper and the lower half-planes, we obtained the modulation of the output signal as a function of 
the phase shift between modes. Using the information of the electromagnetic fields distribution, we calculated the confinement factors for both the fundamental and first order modes which correspond to 0.94 and 0.67 , respectively. These values indicate that the fundamental mode is less sensitive to the upper cladding layer parameters, as more energy is located inside the core of the waveguide than for the first order mode.

Once defined the waveguides, the wafer was covered by a silicon dioxide (refractive index 1.48 at $633 \mathrm{~nm}$ ) cladding layer deposited by PECVD technique. A sensing window was etched in the bimodal part of the waveguide with an area of $15 \times 0.05 \mathrm{~mm}^{2}$ by wet etching of the cladding. The sensing area allows access to the waveguide for sensing evaluation.

The total length of the waveguide (chip) including both single and bimodal parts was $30 \mathrm{~mm}$. The total length of the bimodal part was $25 \mathrm{~mm}$, which included the sensing window with a length of $15 \mathrm{~mm}$, placed $5 \mathrm{~mm}$ off the chip exit. The chip was $30 \times 10 \mathrm{~mm}^{2}$ in size and contained 16 independent interferometers divided in groups of four. As 12 chips are produced, a total number of 192 devices are available per wafer.

Polishing of the chip edges was done by the consecutively use of sandpapers with different grain size $(9,3$ and $0.3 \mu \mathrm{m}$ grain size) with a Polishing Machine (Logitech CL50) until an optical quality for end-coupling was achieved (rugosity of 0.3 $\mu \mathrm{m})$.

Cleaning of the chip was performed by flushing acetone, ethanol and water and drying with $\mathrm{N} 2$ before evaluation.

\section{B. Evaluation setup}

TE light was coupled from a He-Ne laser $(\lambda=632.8 \mathrm{~nm}, 15$ $\mathrm{mW}$ ) into the waveguide by using a microscope objective $(40 x)$. The interference pattern at the output of the bimodal waveguide was monitored using a two sectional photodiode (S5870, Hamamtsu) and each section was connected to a current amplifier (PDA 200C, Thorlabs).

Light exiting the waveguide generates currents $I_{u p}$, and $I_{d o w n}$ in the upper and the lower sections of the photodetector, respectively. The values of the currents were used for calculating the parameter $S$ (sensitivity), according with the expression:

$$
S=\frac{I_{u p}-I_{\text {down }}}{I_{u p}+I_{\text {down }}}
$$

For liquid measurements, a flow cell was designed and fabricated in PDMS. The microfluidic header has four independent flow channels ( $3 \mu$ l volume each) which cover each of the sensing areas of a group of four interferometers. The microfluidic header is coupled to a syringe pump (New Era Pump Systems, Inc) and an injection valve (Idex, US) and allows the sequential evaluation of up to 4 sensors per chip. The flow cell is easily attached and detached to the chip surface and a hermetic sealing is ensured by screwing the cell to the chip support.

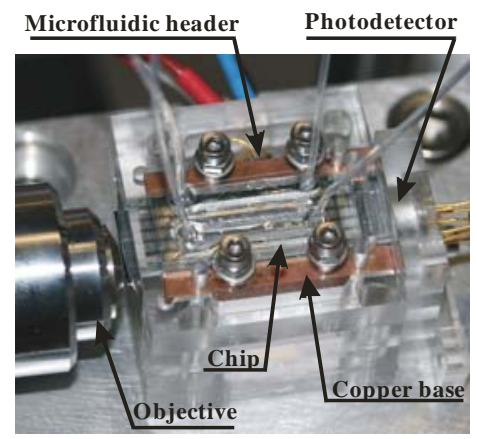

Fig. 3. Photograph of the chip mounted into the temperature stabilization unit and covered by the microfluidic header.

The photodetector is directly attached to the chip output facet. The chip was installed on a copper base mounted over a thermoelectric element (TEC1.4-6, Thorlabs) connected to a Temperature Controller (TED 200C, Thorlabs). The system provided temperature stabilization of the chip with 0.01 degrees accuracy. A photo of the chip mounted in the setup is shown in Fig. 3.

Using a temperature change of one degree we can monitor the intermodal phase difference of the device. For this evaluation the sensing area was covered with a polymer with refractive index of 1.42. The change in the intermodal phase difference induced by temperature variation is shown in Fig. 4. As can be observed, a linear reduction of the total output signal by $45 \%$ was obtained (dash line on Fig. 4). This influenced the coupling efficiency, but did not affect the modulation depth of the output signal: the phase difference linearly increases with the temperature (solid line on Fig. 4).

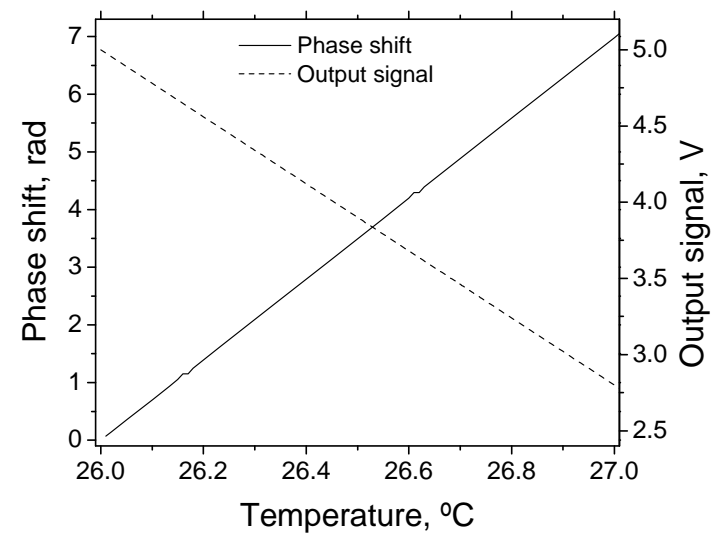

Fig. 4. Response of the sensor to temperature changes, monitored as a change of the phase difference between modes. It can be also observed the influence of the temperature on the total output signal due to the thermal expansion of the chip holder.

The output signal is the sum of the signals obtained after the current-to-voltage conversion of the currents generated by both photodiode sections. The temperature $T$ and the parameter $S$ have been registered simultaneously in this experiment. The function $S(T)$ was approximated by an harmonic function and it was recalculated into the phase shift 
using the expression:

$$
\varphi(T)=\sum_{i=1}^{T / \Delta T} \sin ^{-1}\left(\frac{S\left(T_{i}\right)}{S_{m}}\right)-\sin ^{-1}\left(\frac{S\left(T_{i-1}\right)}{S_{m}}\right)
$$

where $\Delta T$ corresponds to the sampling interval of $S(T)$ and $S_{m}$ is the amplitude of $S(T)$.

The same chip with the polymer sealing the sensing area was used for testing the drift in the setup. The temperature was maintained with stability of 0.01 degree, while the fluctuation of the $S$ parameter did not exceed $2 \%$ during 15 minutes.

\section{HOMOGENEOUS SENSING}

In order to check the sensitivity of our device, a sensitivity calibration evaluating refractive index changes was carried out. Using the microfluidic system described above we performed injections of different concentrations of $\mathrm{HCl}$ $(0.2 \mathrm{M}, 0.1 \mathrm{M}, 0.05 \mathrm{M}$ and $0.025 \mathrm{M})$. Previously to injection, the refractive index of the solutions was checked with an ABBE Refractometer (Optic Ivymen System, Spain). Refractive indexes of the $\mathrm{HCl}$ solutions are summarized in Table 1 .

TABLE I

VALUES OF THE ABSOLUTE REFRACTIVE INDEX (N) OF HCL CONCENTRATIONS AND CHANGE IN REFRACTIVE INDEX $(\Delta \mathrm{N})$

\begin{tabular}{ccc}
\hline \hline HCl concentration & $\mathrm{n}$ & $\Delta \mathrm{n}$ \\
\hline 0.2 & 1.3342 & $1.9 \mathrm{E}-3$ \\
0.1 & 1.3333 & $1 \mathrm{E}-3$ \\
0.05 & 1.3331 & $6 \mathrm{E}-4$ \\
0.025 & 1.3329 & $4 \mathrm{E}-4$ \\
\hline \hline
\end{tabular}

In the experiments, we maintain a continuous water flow over the sensor surface which was gradually replaced by an injected loop of $250 \mu \mathrm{l}$ of the $\mathrm{HCl}$ solution under evaluation. Changes of the refractive index over the sensor area induce a phase change through the evanescent field interaction.

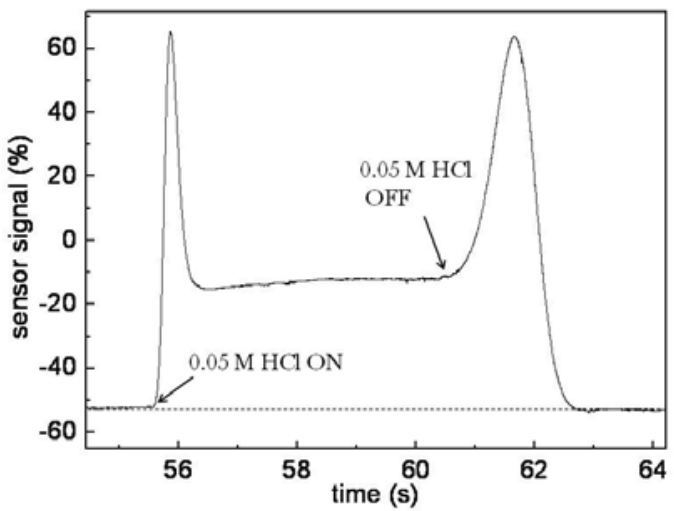

Figure 5: Real time monitoring of the phase change due to the injection of 250 $\mu \mathrm{L}$ of $\mathrm{HCl} 0.05 \mathrm{M}$ over the sensor surface.

As an example, Figure 5 shows the real-time phase change when a concentration of $0.05 \mathrm{M}$ of $\mathrm{HCl}(\mathrm{n}=1.3327)$ was injected. The change in the bulk refractive index $\left(\Delta \mathrm{n}=5.9 \times 10^{-4}\right.$ RIU) produces a phase change which is monitored since $\mathrm{HCl}$ arrives to the sensor area until it goes out due to the pump flow; sensor area is filled again with water and baseline is recovered (dashed line in the figure).

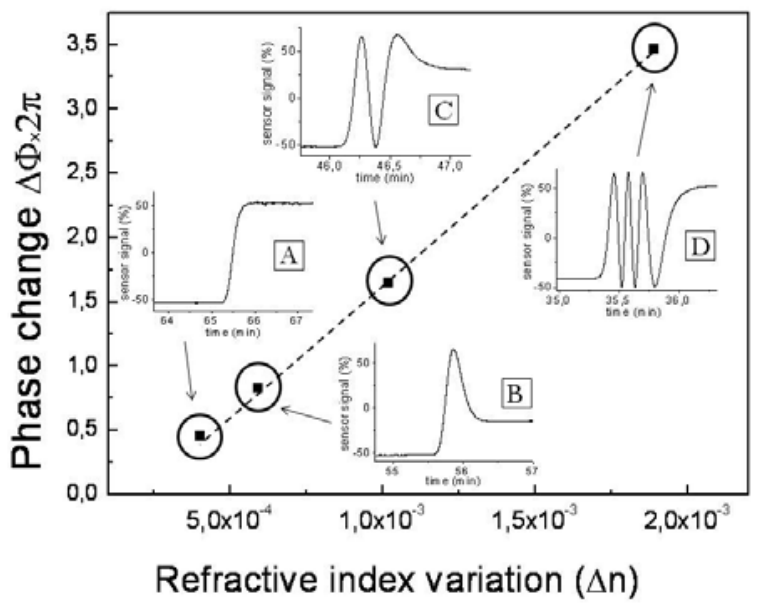

Figure 6: Sensitivity evaluation of the BiMW sensor: phase change is evaluated as a function of the refractive index variation due to the injected $\mathrm{HCl}$ concentrations. Inset: Figure A) detection of $\mathrm{HCl} 0.025 \mathrm{M}$ corresponding to a phase change of $0.45 \times 2 \pi \mathrm{rad}$, Figure B) detection of $\mathrm{HCl} 0.05 \mathrm{M}$ corresponding to a phase change of $0.83 \times 2 \pi \mathrm{rad}$, Figure C) detection of $\mathrm{HCl}$ $0.1 \mathrm{M}$ corresponding to a phase change of $1.65 \times 2 \pi$ rad, Figure D) detection of $\mathrm{HCl} 0.2 \mathrm{M}$ corresponding to a phase change of $3.47 \times 2 \pi \mathrm{rad}$ (in all the insets only $\mathrm{HCl}$ entrance is showed).

Phase variation is plotted versus index variation $(\Delta \mathrm{n})$ and the experimental sensitivity is determine as $d(\Phi \cdot 2 \pi) / d n=$ 2026. As the lowest phase shift which could be evaluated is considered to be three times the $\mathrm{S} / \mathrm{N}$ ratio which is $5 \times 10^{-4}$ $2 \pi \mathrm{rad}$, this means that a theoretical detection limit of $\Delta n_{\min }=2.5 \times 10^{-7} \mathrm{RIU}$ could be achieved, corresponding to a surface sensitivity of $3 \times 10^{-5} \mathrm{~nm}^{-1}$ [1].

\section{SURFACE SENSING}

Bovine Serum Albumin (BSA) protein and its specific antibody anti-BSA have been commonly used as a test biomolecular system to demonstrate the applicability of novel waveguide devices as label-free biosensors [11-13]. Acetone, ethanol, Phosphate Buffer Saline (PBS; $10 \mathrm{mM}$ phosphate, $2.9 \mathrm{mM} \mathrm{KCl}, 137 \mathrm{mM} \mathrm{NaCl}, \mathrm{pH}$ 7.4), albumin bovine $95-99 \%$ (BSA) and monoclonal anti-bovine serum albumin antibody (mAb-BSA) produced in mouse were purchased from Sigma. The employed water was Milli-Q grade (Millipore).

Physical adsorption of BSA protein onto the sensor area of the BiMW was done by flowing $250 \mu \mathrm{L}$ of a PBS solution with $50 \mu \mathrm{g} / \mathrm{mL}$ BSA, at a flow rate of $10 \mu \mathrm{L} / \mathrm{min}$. Phase variation $(7.86 \times 2 \pi \mathrm{rad})$ due to the refractive index bulk change by physical adsorption of the protein is observed in Fig. 7. The phase change becomes slower until reaches a stable signal, which means that the surface is totally saturated with proteins. Next, the water flow induces 
the detachment of non-adsorbed protein $(0,15 \times 2 \pi \mathrm{rad})$. Therefore, the total phase change due to receptor attachment was $4.41 \times 2 \pi \mathrm{rad}$, corresponding to a final covering of $0.42 \mathrm{ng} / \mathrm{mm}^{2}[14]$ of BSA receptors.

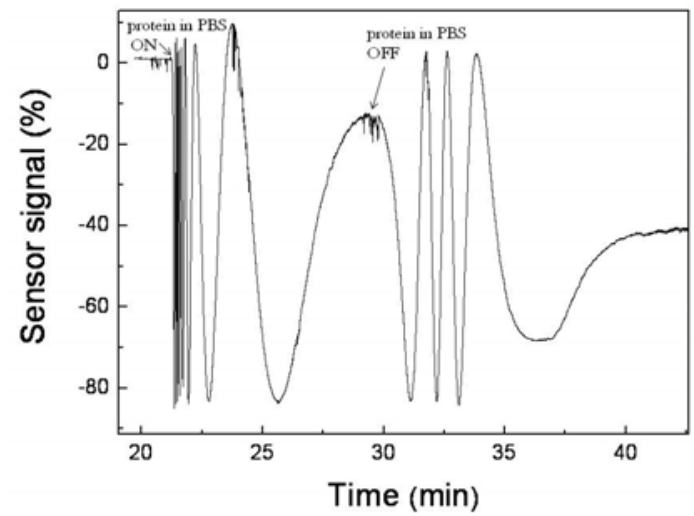

Fig. 7. Real time monitoring of the phase change due to the injection of 250 $\mu \mathrm{L}$ of $50 \mu \mathrm{g} / \mathrm{mL}$ BSA in PBS The observed $\Delta \phi(7.86 \times 2 \pi \mathrm{rad})$ is due to a bulk refractive index change from water to PBS and for the protein adsorption in the sensor area. When the solution of proteins is over, a $\Delta \phi$ of $3.30 \times 2 \pi \mathrm{rad}$ is observed, due to the refractive index change from PBS to water. Total adsorption of protein corresponds to a $\Delta \phi$ of $4.41 \times 2 \pi$ rad. Later, water flow induces the detachment of non-adsorbed protein $(0.15 \times 2 \pi \mathrm{rad})$.

The biomolecular interaction with the specific antibody was accomplished by flowing over the sensor area a solution of 5 $\mu \mathrm{g} / \mathrm{mL}$ of $\mathrm{mAb}-\mathrm{BSA}$ in PBS at a flow rate of $20 \mu \mathrm{L} / \mathrm{min}$, while maintaining a PBS flow (see Fig. 8). Previously water was substituted by PBS as flow buffer (see Inset in Fig. 8). In this case, the $\Delta \phi(0.88 \times 2 \pi \mathrm{rad})$ is only due to the biospecific interaction as buffers employed are the same. Even if the employed concentration of the antibody is high, the results show the excellent performance of our new device for biosensing. A complete analytical characterization of the device is on-going.

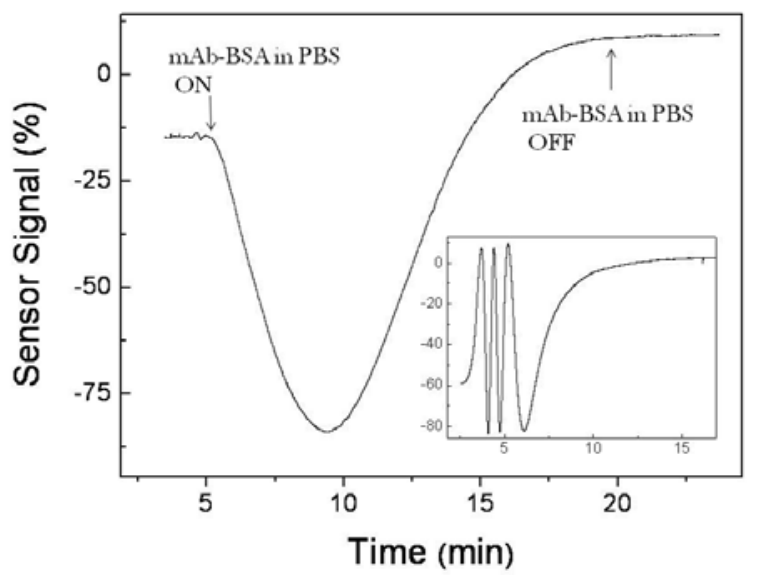

Fig. 8. Real time $\Delta \phi(0.88 \times 2 \pi \mathrm{rad})$ monitoring due to specific interaction of mAb-BSA with modified BSA surface. In the Inset, $\Delta \phi$ due to previous change from water to PBS.

\section{CONCLUSION}

We have presented the optical and biosensing characterization of a simple evanescent field sensor based on a bimodal waveguide interferometer. We have shown that the interferometer is stable enough to be implemented as a biosensor; the sensitivity is independent of the light coupling efficiency and, moreover, the device is easy to be operated by non-experienced users. The problem of the strong temperature dependence is solved by thermal stabilization of the chip. At the same time the temperature control can be used as a test technique for the interferometer real time characterization. We have demonstrated that the device has a high sensitivity $\left(2.5 \times 10^{-7} \mathrm{RIU}\right)$ for label-free sensing. The relatively simple design, high integration level, well-known read out techniques, and high sensitivity made these devices very attractive for further integration in point-of-care instruments.

Further work is being carried out for developing a complete lab-on-a-chip microsystem by integrating the bimodal sensors, a microfluidics network, a phase modulation system to convert the periodic interferometric signals into direct phase measurements, and grating couplers for the in and out coupling of the light.

\section{ACKNOWLEDGEMENTS}

Authors acknowledge the financial support from Fundación M. Botín and the SENA project (TSI-020301-2008-11) from the Spanish Ministry of Industry.

\section{REFERENCES}

[1] F. Prieto, B. Sepúlveda, A. Calle, A. Llobera, C. Domínguez, A. Abad, A. Montoya and L. M. Lechuga, "An integrated optical interferometric nanodevice based on silicon technology for biosensor applications," Nanotechnology, 14, 907-912, 2003.

[2] J. Homola, Surface Plasmon Resonance Sensors for Detection of Chemical and Biological Species, Chem. Rev., 2008, 108 (2), 462-493

[3] A. Ymeti, J. S. Kanger, R. Wijn, P. V. Lambeck, J. Greve, "Development of a multichannel integrated interferometer sensor," Sensors and Actuators B, 83 (2002) 1-7

[4] W. Lukosz and CH Stamm, "Integrated optical interferometer as relative humidity sensor and differential refractometer," Sensors and Actuators A, 25-27 (1991) 185-188

[5] N. Kato, L. Lee, R. Chandrawati, A. P. R. Johnston, and F. Caruso, "Optically characterized DNA multilayered assemblies and phenomenological modeling of layer-by-layer hybridization," J. Phys. Chem. C, 113 (2009) 21185-21195

[6] M. Lohmeyer, L. Wilkens, O. Zhuromsky, H. Dötsch, and P. Herte "Integrated magnetooptic cross strip isolator," Optics Communications, vol. 189, no. 4-6, pp. 251-259, 2001

[7] A. Irace and G. Breglio, "All-silicon optical temperature sensor based on Multi-Mode Interference," Optics Express, vol. 11, no. 22, pp. 28072812, 2003

[8] R. Levy and S. Ruschin, "Design of a single-channel modal interferometer waveguide sensor," IEEE Sensors, vol. 9, no. 2, pp. 146153,2009

[9] K. Zinoviev, L. M. Lechuga, C. Dominguez, "Silicon nitride bimodal waveguides for high sensitivity biosensors," (ECIO) 2010, Proc. European Conference on Optical Integration (ECIO) 2010, Cambridge, UK, WeP40

[10] Patent PCT/ES08/070142, 12/669307 (US)

[11] D. Dorfner, T. Zabel, T. Hürlimann, N. Hauke, L. Frandsen, U. Rant, G. Abstreiter and J. Finley, "Photonic crystal nanostructures for optical biosensing applications," Biosensors and Bioelectronics, vol. 24, pp. $3688-3692,2009$ 
[12] S. J. Choo, B.-C. Lee, S.-M. Lee, J. H. Park and H.-J. Shin Optimization of silicon oxynitrides by plasma-enhanced chemical vapor deposition for an interferometric biosensor. J. Micromech. Microeng. vol. 19, 095007 (9pp), 2009.

[13] C. A. Barrios, M. J. Bañuls, V. González-Pedro, K. B. Gylfason, B. Sánchez, A. Griol, A. Maquieira, H. Sohlström, M. Holgado and R. Casquel. "Label-free optical biosensing with slot-waveguides," Optics Letters, vol. 33, no. 7, pp. 708-710, 2008

[14] N. J. Freeman, L. L. Peel, M. J. Swann, G. H. Cross, A. Reeves, S. Brand and J. R Lu, "Real time, high resolution studies of protein adsorption and structure at solid-liquid interface using dual polarization interferometry," J. Phys.; Condens. Matter, vol. 16, pp. S2493-S2496, 2004

Kirill Zinoviev received Ph.D. degree in engineering from Moscow State Academy of Engineering, Russia, in 1997. He is currently with the Group of Chemical Transducers at Barcelona Institute for Microelectronics, Spain. His research interests are focused on design and fabrication of micro-optical systems.

Ana Belén Gonzalez-Guerrero is working on her $\mathrm{PhD}$ thesis in the Nanobiosensors and Bioanalytical applications Group at the Research Center on Nanoscience and Nanotechnology (CIN2), CSIC. Her research area is focused in the development of evanescent wave biosensors for biological applications.

Carlos Dominguez received $\mathrm{PhD}$ in Chemistry by the University Complutense of Madrid in 1985. He is an expert on optoelectronics technology and Clean Room processing of sensor devices. His current research interests are focused on the topics: technological development for the implementation of MEMS/NEMS integrated in silicon, polymers for packaging and optical elements, MOEMS

Laura M. Lechuga is Full Professor of the Spanish National Research Council (CSIC). She is the Head of the Nanobiosensors and Bioanalytical applications Group at the Research Center on Nanoscience and Nanotechnology (CIN2), CSIC. Her main research areas are the development of biosensor devices based on plasmonics, magnetoplasmonics,integrated photonics and nanomechanics principles, including surface biofunctionalization, microfluidics and lab-on-a-chip integration. The biosensing platforms are applied in the environmental control of pollutants, early diagnosis of cancer and diseases and genomics and proteomics. 